\title{
THE PRODUCT OF TWO ULTRASPHERICAL POLYNOMIALS
}

\author{
by L. CARLITZ*
}

(Received 25 October, 1960)

1. Let

It is familiar that

$$
\sum_{n=0}^{\infty} C_{n}^{v}(x) t^{n}=\left(1-2 x t+t^{2}\right)^{-v}
$$

$$
\sum_{n=0}^{\infty} C_{n}^{v}(x) \frac{t^{n}}{(2 v)_{n}}=\Gamma\left(v+\frac{1}{2}\right) e^{x t}\left\{\frac{1}{2} t\left(1-x^{2}\right)^{\frac{1}{2}}\right\} J_{v-\frac{1}{2}}\left\{t\left(1-x^{2}\right)^{\frac{1}{2}}\right\} .
$$

In the addition theorem $[3$, p. 363]

where

$$
\frac{J_{v}(w)}{\left(\frac{1}{2} w\right)^{v}}=\Gamma(v) \sum_{n=0}^{\infty}(v+n) \frac{J_{v+n}(t)}{\left(\frac{1}{2} t\right)^{v}} \frac{J_{v+n}(z)}{\left(\frac{1}{2} z\right)} C_{n}^{v}(\cos \theta),
$$

$$
w=\left(t^{2}+z^{2}-2 t z \cos \theta\right)^{\frac{1}{2}},
$$

take $\theta=\pi$ and replace $v$ by $v-\frac{1}{2}$. Since

we get

$$
C_{n}^{v-\frac{1}{2}}(-1)=(-1)^{n} \frac{(2 v-1)_{n}}{n !}
$$

$$
\frac{J_{v-\frac{1}{2}}(t+z)}{\left\{\frac{1}{2}(t+z)\right\}^{v-\frac{1}{2}}}=\Gamma\left(v-\frac{1}{2}\right) \sum_{n=0}^{\infty}(-1)^{n}\left(v-\frac{1}{2}+n\right) \frac{(2 v-1)_{n}}{n !} \frac{J_{v-\frac{1}{2}+n}(t)}{\left(\frac{1}{2} t\right)^{v-\frac{1}{2}}} \frac{J_{v-\frac{1}{2}+n}(z)}{\left(\frac{1}{2} z\right)^{v-\frac{1}{2}}} .
$$

We now replace $t$ and $z$ by $t\left(1-x^{2}\right)^{\frac{1}{2}}$ and $z\left(1-x^{2}\right)^{\frac{1}{2}}$, respectively, and use (1). The result is $\sum_{k=0}^{\infty} C_{k}^{v}(x) \frac{(t+z)_{v}^{k}}{(2 v)_{k}}=\sum_{r=0}^{\infty}(-1)^{r} \frac{v-\frac{1}{2}+r}{v-\frac{1}{2}} \frac{(2 v-1)_{r}\left(1-x^{2}\right)^{r}}{r !\left\{\left(v+\frac{1}{2}\right)_{r}\right\}^{2}}$

$$
\times\left(\frac{1}{4} t z\right)^{r} \sum_{m=0}^{\infty} C_{m}^{v+r}(x) \frac{t^{m}}{(2 v+2 r)_{m}} \sum_{n=0}^{\infty} C_{n}^{v+r}(x) \frac{z^{n}}{(2 v+2 r)_{n}}
$$

Comparing coefficients of $t^{m} z^{n}$ on both sides, we get, for $v \neq \frac{1}{2}$,

$$
\left(\begin{array}{c}
m+n \\
m
\end{array}\right) \frac{C_{m+n}^{v}(x)}{(2 v)_{m+n}}=\sum_{r=0}^{\min (m, n)}\left(-\frac{1}{4}\right)^{r} \frac{v-\frac{1}{2}+r}{v-\frac{1}{2}} \frac{(2 v-1)_{r}}{r !} \frac{\left(1-x^{2}\right)^{r}}{\left\{\left(v+\frac{1}{2}\right)_{r}\right\}^{2}} \frac{C_{m-r}^{v+r}(x) C_{n-r}^{v+r}(x)}{(2 v+2 r)_{m-r}(2 v+2 r)_{n-r}}
$$

For $v=\frac{1}{2}$ we have, however,

$$
P_{m+n}(x)=P_{m}(x) P_{n}(x)+2 . m ! n ! \sum_{r=1}^{\min (m, n)}\left(-\frac{1}{4}\right)^{r} \frac{\left(1-x^{2}\right)^{r}}{r ! r !} \frac{C_{m-r}^{\frac{1}{2}+r}(x)}{(2 r+1)_{m-r}} \frac{C_{n-r}^{\frac{1}{2}+r}(x)}{(2 r+1)_{n-r}},
$$

where $P_{n}(x)$ is the Legendre polynomial. Since $[4$, p. 329]

(4) may be written as

$$
C_{n-r}^{\frac{1}{2}+r}(x)=\frac{\left(x^{2}-1\right)^{-\frac{1}{2} r}}{2^{r}\left(\frac{1}{2}\right)_{r}} P_{n}^{r}(x)
$$

$$
P_{m+n}(x)=P_{m}(x) P_{n}(x)+2 \sum_{r=1}^{\min (m, n)} \frac{P_{m}^{r}(x) P_{n}^{r}(x)}{(m+1)_{r}(n+1)_{r}}
$$

* Supported in part by National Science Foundation grant G-9425. 
By taking $v=q+\frac{1}{2}$ in (3) we get the identity

$$
\left(\begin{array}{c}
m+n \\
m
\end{array}\right) \frac{P_{m+n+q}^{q}(x)}{q !(2 q+1)_{m+n}}=\left(x^{2}-1\right)^{ \pm q} \sum_{r=0}^{\min (m, n)} \frac{(2 q+r) !}{r !} \frac{P_{m}^{q+r}(x) P_{n}^{q+r}(x)}{(m+2 q+r) !(n+2 q+r) !} \quad(q \geqq 1) .
$$

2. To invert (3) we require the formula

$$
\sum_{r=0}^{\infty} \frac{1}{\Gamma(v+r+1)} \frac{(2 v+r+1)_{r}}{r !} \frac{J_{v+r}(t+z)}{\left\{\frac{1}{2}(t+z)\right\}^{v+r}}\left(\frac{1}{4} t z\right)^{r}=\frac{J_{v}(t)}{\left(\frac{1}{2} t\right)^{v}} \frac{J_{v}(z)}{\left(\frac{1}{2} z\right)^{v}} .
$$

Indeed, on making use of (2), it is clear that the left member of (7) is equal to

$$
\begin{aligned}
\sum_{r=0}^{\infty} \frac{v}{v+r} \frac{(2 v+r+1)_{r}}{r !} \sum_{s=0}^{\infty}(-1)^{s}(v+r+s) \frac{(2 v+2 r)_{s} J_{v+r+s}(t)}{s !\left(\frac{1}{2} t\right)^{v}} \frac{J_{v+r+s}(z)}{\left(\frac{1}{2} z\right)^{v}} \\
=\sum_{n=0}^{\infty}(v+n) \frac{J_{v+n}(t)}{\left(\frac{1}{2} t\right)^{v}} \frac{J_{v+n}(z)}{\left(\frac{1}{2} z\right)^{v}} \cdot \sum_{r+s=n} \frac{(-1)^{s}}{v+r} \frac{(2 v+r+1)_{r}(2 v+2 r)_{s} .}{r ! s !}
\end{aligned}
$$

Now, for $n \geqq 1$ the inner sum is equal to

$$
\sum_{r=0}^{n} \frac{(-1)^{n-r}}{-r} \frac{(2 v+r+1)_{r}(2 v+2 r+1)_{s-1}(2 v+2 r)}{r ! s !}=\frac{2}{n !} \sum_{r=0}^{n}(-1)^{n-r}\left(\begin{array}{l}
n \\
r
\end{array}\right)(2 v+r+1)_{n-1}=0,
$$

since the $n$th difference of a polynomial of degree $n-1$ vanishes. For $n=0$, on the other hand, the inner sum is $1 / v$. This evidently proves (7). Note that the formula holds for $v=0$.

We now replace $v$ by $v-\frac{1}{2}, t$ and $z$ by $t\left(1-x^{2}\right)^{\frac{1}{2}}$ and $z\left(1-x^{2}\right)^{\frac{1}{2}}$, respectively, and again use (1). Then

$$
\sum_{m=0}^{\infty} C_{m}^{v}(x) \frac{t^{m}}{(2 v)_{m}} \sum_{n=0}^{\infty} C_{n}^{v}(x) \frac{z^{n}}{(2 v)_{n}}=\sum_{r=0}^{\infty} \frac{(2 v+r)_{r}}{r !\left(v+\frac{1}{2}\right)_{r}\left(v+\frac{1}{2}\right)_{r}}\left(\frac{1}{t} t z\right)^{r}\left(1-x^{2}\right)^{r} \sum_{n=0}^{\infty} C_{n}^{v+r}(x) \frac{(t-z)^{n}}{(2 v+2 r)_{n}} .
$$

Equating coefficients we get

$$
\frac{C_{m}^{v}(x)}{(2 v)_{m}} \frac{C_{n}^{v}(x)}{(2 v)_{n}}=\sum_{r=0}^{\min (m, n)} \frac{(2 v+r)_{r}}{r !\left(v+\frac{1}{2}\right)_{r}\left(v+\frac{1}{2}\right)_{r}}\left(\begin{array}{c}
m+n-2 r \\
m-r
\end{array}\right) \frac{\left(1-x^{2}\right)^{r}}{4^{r}} \frac{C_{m+n-2 r}^{v+r}(x)}{(2 v+2 r)_{m+n-2 r}}
$$

or, if we prefer,

$$
\begin{aligned}
C_{m}^{v}(x) C_{n}^{v}(x)=\frac{(2 v)_{m}(2 v)_{n}}{(2 v)_{m+n}} & \sum_{r=0}^{\min (m, n)}\left(\begin{array}{c}
m+n-2 r \\
m-r
\end{array}\right) \frac{(2 v+r)_{r}(v)_{r}}{r !\left(v+\frac{1}{2}\right)_{r}}\left(1-x^{2}\right)^{r} C_{m+n-2 r}^{v+r}(x) \\
& =\frac{(2 v)_{m}(2 v)_{n}}{(2 v)_{m+n}} \sum_{r=0}^{\min (m, n)}\left(\begin{array}{c}
m+n-2 r \\
m-r
\end{array}\right) \frac{(v)_{r}(v)_{r}}{r !(2 v)_{r}} 4^{r}\left(1-x^{2}\right) C_{m+n-2 r}^{v+r}(x) .
\end{aligned}
$$

In particular, for $v=\frac{1}{2}$, (8) becomes

$$
\left(\begin{array}{c}
m+n \\
m
\end{array}\right) P_{m}(x) P_{n}(x)=\sum_{r=0}^{\min (m, n)}\left(\begin{array}{c}
m+n-2 r \\
m-r
\end{array}\right) \frac{(2 r) !}{(r !)^{3}} \frac{\left(x^{2}-1\right)^{\frac{1}{2} r}}{2^{r}} P_{m+n-r}^{r}(x) .
$$

More generally for $v=q+\frac{1}{2}$ we get

$$
\begin{aligned}
\frac{(2 q+1)_{m-n}}{(2 q+1)_{m}(2 q+1)_{n}} P_{m+q}^{q}(x) P_{n+q}^{q}(x)=(2 q) ! & \sum_{r=0}^{\min (m, n)}(-1)^{r}\left(\begin{array}{c}
m+n-2 r \\
m-r
\end{array}\right) \\
& \times \frac{(2 q+2 r) !}{r !(q+r) !(2 q+r) !} \frac{\left(x^{2}-1\right)^{t(q+r)}}{2^{q+r}} P_{m+n+q-r}^{q+r}(x) .
\end{aligned}
$$


3. Note that (10) differs from the formula found by Bailey [2] for the product of associated Legendre polynomials. Similarly (6) differs from the inverse formula found by Al-Salam [1]. However we shall now show that (10) does indeed imply Bailey's identity.

We recall that

$$
4 v(n+v-1)\left(1-x^{2}\right) C_{n-2}^{v+1}(x)=(n+2 v-1)(n+2 v-2) C_{n-2}^{v}(x)-n(n-1) C_{n}^{v}(x) .
$$

We shall show that generally

$$
\frac{4^{r}(v)_{r}(n-2 r) !}{(2 v)_{n}}\left(1-x^{2}\right)^{r} C_{n-2 r}^{v+r}(x)=\sum_{s=0}^{r}(-1)^{r-s}\left(\begin{array}{l}
r \\
s
\end{array}\right) \frac{n+v-2 s}{(n+v-r-s)_{r+1}} \frac{(n-2 s) !}{(2 v)_{n-2 s}} C_{n-2 s}^{v}(x),
$$

for $2 r \leqq n$. For $r=1,(12)$ evidently reduces to (11). Now assuming that (12) holds for the value $r$, we get (replacing $n$ by $n-2$ and $v$ by $v+1$ )

$$
\begin{aligned}
& \frac{4^{r+1}(v)_{r+1}(n-2 r-2) !}{(2 v)_{n}}\left(1-x^{2}\right)^{r+1} C_{n-2 r-2}^{v+r+1}(x) \\
& =\sum_{s=0}^{r}(-1)^{r-s}\left(\begin{array}{l}
r \\
s
\end{array}\right) \frac{(n-2 s-2) !}{(n+v-r-s-1)_{r+1}(2 v)_{n-2 s}} 4 v(n+v-2 s-1)\left(1-x^{2}\right) C_{n-2 s-2}^{v+1}(x) \\
& =\sum_{s=0}^{r}(-1)^{r-s}\left(\begin{array}{l}
r \\
s
\end{array}\right) \frac{(n-2 s-2) !}{(n+v-r-s-1)_{r+1}(2 v)_{n-2 s}} \\
& \times\left\{(n+2 v-2 s-1)(n+2 v-2 s-2) C_{n-2 s-2}^{v}(x)-(n-2 s)(n-2 s-1) C_{n-2 s}^{v}(x)\right\} \\
& =\sum_{s=0}^{r+1}(-1)^{r+1-s}\left\{\left(\begin{array}{c}
r \\
s-1
\end{array}\right) \frac{1}{(n+v-r-s)_{r+1}}+\left(\begin{array}{l}
r \\
s
\end{array}\right) \frac{1}{(n+v-r-s-1)_{r+1}}\right\} \frac{(n-2 s) !}{(2 v)_{n-2 s}} C_{n-2 s}^{v}(x) \\
& =\sum_{s=0}^{r+1}(-1)^{r+1-s}\left(\begin{array}{c}
r+1 \\
s
\end{array}\right) \frac{n+v-2 s}{(n+v-r-s-1)_{r+2}} \frac{(n-2 s) !}{(2 v)_{n-2 s}} C_{n-2 s}^{v}(x),
\end{aligned}
$$

so that (12) holds for the value $r+1$.

We remark that

$$
\frac{\left(v+\frac{1}{2}\right)_{r}(n+1)_{2 r}}{(2 v)_{n+2 r}} C_{n+2 r}^{v}(x)=\sum_{s=0}^{r}(-1)^{s}\left(\begin{array}{l}
r \\
s
\end{array}\right) \frac{(n+v+r)^{s}}{(2 v+2 s)_{n}}\left(1-x^{2}\right)^{s} C_{n}^{v+s}(x),
$$

which is the inverse of (12), can also be proved by induction with respect to $r$.

4. Returning to (8) and making use of (12), we get

$$
\begin{gathered}
C_{m}^{v}(x) C_{n}^{v}(x)=(2 v)_{m}(2 v)_{n} \sum_{r=0}^{\min (m, n)}\left(\begin{array}{c}
m+n-2 r \\
m-r
\end{array}\right) \frac{(2 v+r)_{r}}{r !\left(v+\frac{1}{2}\right)_{r}} \\
\times \frac{4^{-r}}{(m+n-2 r) !} \sum_{s=0}^{r}(-1)^{r-s}\left(\begin{array}{l}
r \\
s
\end{array}\right) \frac{m+n+v-2 s}{(m+r+v-r-s)_{r+1}} \frac{(m+n-2 s) !}{(2 v)_{m+n-2 s}} C_{m+n-2 s}^{v}(x) \\
=(2 v)_{m}(2 v)_{n} \sum_{s=0}^{m i n(m, n)} \frac{m+n+v-2 s}{m+n+v-s} \frac{(m+n-2 s) !}{(2 v)_{m+n-2 s}} C_{m+n-2 s}^{v}(x) \\
\times \sum_{r=s}^{\min (m, n)}(-1)^{r-s}\left(\begin{array}{l}
r \\
s
\end{array}\right) \frac{(v)_{r}}{r !(m-r) !(n-r) !(2 v)_{r}(m+n+v-r-s)_{r}} .
\end{gathered}
$$


The inner sum is equal to

$$
\begin{gathered}
\frac{(v)_{s}}{s !(m-s) !(n-s) !(2 v)_{s}(m+n+v-2 s)_{s}} \sum_{r=0}^{\min (m-s, n-s)} \frac{(-m+s)_{r}(-n+s)_{r}(v+s)_{r}}{r !(2 v+s)_{r}(1+2 s-m-n-v)_{r}} \\
=\frac{(v)_{s}}{s !(m-s) !(n-s) !(2 v)_{s}(m+n+v-2 s)_{s}}{ }_{3} F_{2}\left[\begin{array}{c}
-m+s,-n+s, v+s \\
2 v+s, 1+2 s-m-n-v
\end{array}\right] \\
=\frac{(v)_{s}}{s !(m-s) !(n-s) !(2 v)_{s}(m+n+v-2 s)_{s}} \frac{(v)_{m-s}(v)_{n-s}(2 v)_{s}(2 v)_{m+n-s}}{(v)_{m+n-2 s}(2 v)_{m}(2 v)_{n}}
\end{gathered}
$$

by Saalschütz's theorem. We therefore get

$$
C_{m}^{v}(x) C_{n}^{v}(x)=\sum_{s=0}^{\min (m, n)} \frac{m+n+v-2 s}{m+n+v-s} \frac{(v)_{s}(v)_{m-s}(v)_{n-s}}{s !(m-s) !(n-s) !} \frac{(2 v)_{m+n-s}}{(v)_{m+n-s}} \frac{(m+n-2 s) !}{(2 v)_{m+n-2 s}} C_{m+n-2 s}^{v}(x) .
$$

For $v=\frac{1}{2}$, (14) reduces to

where

$$
P_{m}(x) P_{n}(x)=\sum_{s=0}^{\min (m, n)} \frac{m+n+\frac{1}{2}-2 s}{m+n+\frac{1}{2}-s} \frac{A_{s} A_{m-s} A_{n-s}}{A_{m+n-s}} P_{m+n-2 s}(x),
$$

$$
A_{r}=\frac{\left(\frac{1}{2}\right)_{r}}{r !}
$$

(15) is the familiar formula of Adams and Neumann. For $v=q+\frac{1}{2}$, (14) becomes

$$
\begin{aligned}
\left(x^{2}-1\right)^{\frac{1}{2} q} P_{m}^{q}(x) P_{n}^{q}(x)=2^{q} \sum_{s=0}^{\min (m-q, n-q)} \frac{m+n-q-2 s+\frac{1}{2}}{m+n-q-s+\frac{1}{2}} \frac{\left(q+\frac{1}{2}\right)_{s}\left(\frac{1}{2}\right)_{m-s}\left(\frac{1}{2}\right)_{n-s}}{s !(m-q-s) !(n-q-s) !} \\
\times \frac{(m+n-s) !}{\left(\frac{1}{2}\right)_{m+n-q-s}} \frac{(m+n-2 q-2 s) !}{(m+n-2 s) !} P_{m+n-q-2 s}^{q}(x),
\end{aligned}
$$

which is in agreement with Bailey's formula.

\section{REFERENCES}

1. W. A. Al-Salam, On the product of two Legendre polynomials, Math. Scand. 4 (1956), 239-242.

2. W. N. Bailey, On the product of two associated Legendre functions, Quart. J. Math. Oxford Ser. (2) 11 (1940), 30-35.

3. G. N. Watson, Theory of Bessel functions (second edn, Cambridge 1944).

4. E. T. Whittaker and G. N. Watson, A course of modern analysis (fourth edn, Cambridge, 1927)

DUKe UNIVERSITY

DURHAM

North Carolina 